\title{
Malaria: It's Gynecological and Obstretic Effects on Humans - A Short Note
}

\author{
Maria A Grácio* and António J Santos Grácio \\ Instituto de Higiene e Medicina Tropical / Universidade Nova de Lisboa, Lisboa, Portugal
}

${ }^{*}$ Corresponding author: Maria A Grácio, Instituto de Higiene e Medicina Tropical / Universidade Nova de Lisboa, Rua da Junqueira 100, 1348-008 Lisboa, Portugal; Email: mameliahelm@ihmt.unl.pt

Received: September 01, 2019; Accepted: September 16, 2019; Published: October 06, 2019;

\section{Short Commentary}

Malaria is a vector borne disease of man caused by protozoans of the genus Plasmodium - P. vivax, P.ovale, P. malariae, P. falciparum and, more recently, $P$. knowlesi [1]. These parasites are present within the red blood cells, and they are transmitted by mosquitoes of the genus Anopheles.

Considering the medical importance of malaria in the context of the gynaecological and obstetric fields we have as objectives in this manuscript to contribute: (i) to the divulgation of the knowledge of human malaria in a general context; (ii) to emphasize the gynaecological and obstetric effects of malaria in the human population. In support of these objectives we present:

In article [2] we emphasized "uncomplicated malaria entails a series of recurring episodes of chills, intense fever, and sweating and often includes other symptoms such as headache, malaise, fatigue, body aches, nauseas, and vomiting. In some cases, and especially in groups, such as children and pregnant women, the disease can progress to "severe malaria," including complications, such as cerebral malaria/coma, seizures, severe anaemia, respiratory distress, kidney and liver failure, cardiovascular collapse, and shock".

This article [3] states that "if a woman gets malaria while pregnant, she and her baby have an increased risk of developing serious complications such as: (1) premature birth - birth before 37 weeks of pregnancy: (i) low birth weight; (ii) restricted growth of the baby in the womb; (2) stillbirth; (3) miscarriage - death of the mother.

\section{Conclusion}

It was here demonstrated that malaria infection can be one cause of human infertility, and of strong negatives effects on pregnant women and their babies. We hope that within a short period of time malaria is combated of sustained form in the world and that it is irradiated soon based, principally, in the initiative of the WHO, Known as the E-2020 initiative and malaria elimination [4].

Keywords: Malaria; Anopheles; pregnancy; gynecology; obstetric; vector-borne diseases.

\section{References}

1. White NJ (2008) Plasmodium knowlesi: the fifth human malaria parasite. Clin Infect Dis. 2008 Jan 15; 46: 172-3.

2. Marrelli MT, Brotto M (2016) The effect of malaria and anti-malarial drugs on skeletal and cardiac muscles. Malaria Journal 15: 524,

3. https://www.nhs.uk/conditions/malaria/complications/

4. Q\&A on the E-2020 initiative and malaria elimination - WHO (3 July 2019).
Citation:

Maria A Grácio and António J Santos Grácio (2019) Malaria: It’s Gynecological and Obstretic Effects on Humans - A Short Note. Integr Gyn Obstet J Volume 2(4): $1-1$. 